\title{
First year engineering mathematics: the London South Bank University experience
}

Cosette Crisan

Centre of Mathematics Education

London South Bank University

crisanc@lsbu.ac.uk
Larissa Fradkin, Department of Engineering, LSBU, fradkil@lsbu.ac.uk

Victor Zernov, Department of Engineering, LSBU, zernovv@lsbu.ac.uk

Steve Lerman, Centre of Mathematics Education, LSBU, lermans@lsbu.ac.uk

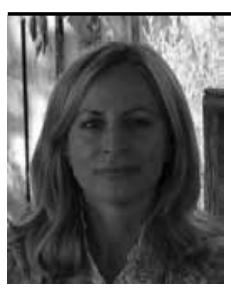

Larissa Fradkin

Department of Electrical Computer and

Communications Engineering

London South Bank University

fradkil@lsbu.ac.uk

(Now at Sound Mathematics Ltd.,

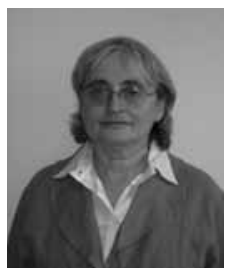

(ambridge)

Victor Zernov

Department of Electrical Computer and

Communications Engineering

London South Bank University

zernovv@lsbu.ac.uk

(Now at Sound Mathematics Ltd.,

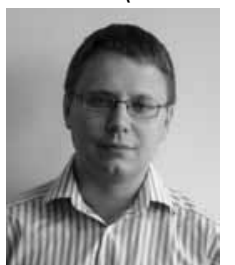

(ambridge)

Steve Lerman

Centre of Mathematics Education

London South Bank University

lermans@lsbu.ac.uk

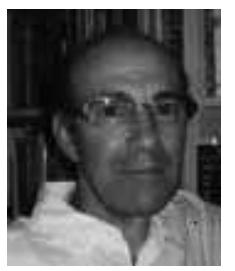

This short article describes an innovative approach to teaching mathematics to first year undergraduates on a variety of B. Eng. courses offered in the Faculty of Engineering, Science and Built Environment (FESBE) of London South Bank University (LSBU).

\section{Introduction}

All over the world undergraduates join science and engineering courses with poorer mathematical background than in the past. The problem is particularly acute in the United Kingdom [7]. University tutors and lecturers spend an inordinate amount of time covering the basics. The FESBE experience shows that students can still achieve deep learning of mathematics - and remarkably quickly - through a teacher-guided (often called Socratic) dialogue, based on the frequent reinforcement of basic mathematical abstractions through Eulerian sequencing. The latter is a systematic approach to mathematics as a language, which allows students to analyse (sequence) given mathematical expressions and thus find the relevant solution algorithm (sequence of solution steps).

\section{Background information}

Many first year FESBE undergraduates study a unit called Introductory Mathematics. The unit provides students with the necessary mathematical tools and methods needed in all other engineering programmes, namely, algebra of numbers, including complex numbers, and calculus. The unit, which is taught over two semesters, is delivered as a two-hour lecture every week. In addition, two-hour tutorials take place once a week.

The minimum entry requirement at $\mathrm{LSBU}$ is $\mathrm{E}$ in A-level mathematics (or equivalent). However, some mature students are accepted even without reaching this level. Also, up to $26 \%$ of all students may be dyslexic, dyspraxic or dyscalculic [6], although most are not aware of their condition. Working with similar student intakes for the past 16 years, a teaching approach had been developed which has been shown time and again to be successful: not only does it lead to the higher pass rate, but it also promotes transferable skills, such as study skills, debating skills, participating in technical discussion and recognising a familiar pattern in an unfamiliar picture.

\section{The FESBE teaching methodology}

As mentioned in the Introduction, the teaching approach under discussion can be classed as Socratic dialogue based on Eulerian sequencing. Originally, the concept of Socratic dialogue has been associated with literary works developed in Greece 
at the turn of the fourth century $B C$, preserved in the Plato dialogues, in which characters discuss moral and philosophical problems, using the Socratic (question and answer) method. Towards the end of the last century, the method was extended by such educationalists as Collins [3], who introduced it into a general pedagogical discourse, and Hake [5], who revolutionised the teaching of undergraduate physics, allowing ordinary learners with few proficiencies in explanatory reasoning to master Newtonian mechanics.

At LSBU, it has been adapted to teaching mathematics to large classes (up to 100 students) of ordinary learners. In this context the "dialogues" typically involve two speakers at any one time, with one (teacher) leading and structuring the discussion. The practice involves a teacher asking a series of questions surrounding a mathematical concept or algorithm, and answering questions posed by students. In addition, the teacher often asks what questions the students should ask themselves to proceed with a problem solution. It is argued that the modern Socratic dialogues should be conducted in a friendly and sometimes humorous manner.

Field notes and audio recordings of lectures conducted by independent researchers (Crisan and Lerman) confirm the interactive nature of both tutorials and lectures, with students being continually encouraged to answer or pose questions and contribute to discussions. The questions asked are either probing, for example, "What would happen to a graph of a function if a constant is added to its argument?" or are of clarification type, such as, "What do we mean by a 'constant?", "What methods of graph sketching graphs have we already learned?", or they probe reasons and implications, such as, "Can you give me an example?", “What are the main features of a parabola?"

In talking to researchers, one student who has a secure mathematics background said that he found the teaching of the topics very different to how he was taught at pre-university level. However, "once you get used to the approach, it is OK; it is mainly the same thing but presented differently".

At the beginning of each lecture, it is suggested that the tutor invites students to do informal concept mapping by asking "What modules are covered in the introductory mathematics?", "What are the main concepts in calculus?", "What type of functions do we study in calculus?", "What operations on functions do we study in calculus?" and "Why do we study calculus?"The students seem comfortable to offer answers to the questions posed. It is further suggested to make sure that all students are engaged in the discussions. This can be done by inviting contributions from both students who volunteer answers and students who do not. Observations and informal chats with the latter group conducted by researchers revealed that they did not feel threatened by this strategy even if they did not always have the answer to the questions asked. This may well be due to the fact that the teacher expectations in terms of students' participation in the learning process are spelled out in the unit guide distributed among students in the beginning of the academic year. Moreover, it is often repeated in class that if students feel unwilling or unable to participate on a particular day all they are asked to do is inform the teacher of this fact before the class commences.

All of the students interviewed by researchers said that they needed time to 'get used' to this teaching approach, saying things like "I was not used to explaining the mathematics". In the past they just "did it", without much verbalising or questioning as to 'why' and 'how'. They agreed that this approach "forced you to think, to really understand the mathematics".

While the Socratic dialogue assures continual student engagement and provides an immediate feedback to both teacher and student [3], the systematic approach to teaching mathematical abstractions to learners of unexceptional ability can be traced back to Euler who believed that any student can be taught and enjoy basic mathematics, and this is the origin of the phrase "Eulerian sequencing". The sequencing consists in making explicit the primary structures of mathematical expressions and ordering the solution steps accordingly. It is in a perfect accord with recent pedagogical findings which suggest that,

"The amount learned is proportional to the number of selfexplanations that a student generates", the self-explanations being comments on a solution step "that contain... domainrelevant information over and above what was stated in the description of the step" [2]. To quote Craig et al. [4], "there are two general sources for self-explanations: The first is deduction from laws, rules, concepts and definitions acquired earlier, usually by simply instantiating a general principle, concept, or procedure with information relevant to the solution step. The second explanation is generalisation and extension of the step." Such construction of the content of the solution step yields new general knowledge that helps complete the students' otherwise incomplete understanding of the domain principles and concepts. The practical outcome is that it allows students to solve not only the problems they repeatedly rehearsed before, but problems that they have never seen. The learners acquire the ability to recognise familiar patterns in unfamiliar pictures, and thus the students are better equipped to solve any questions. 
To illustrate Socratic questioning via Eulerian sequencing in the context of differentiation, the emphasis is on making certain the students can confidently use concepts, such as an independent variable, dependent variable, differentiation variable, function, constant and can identify the corresponding entities in the given mathematical expression. The term function is particularly interesting in that at the school level it is mainly used to denote a dependent variable, while its broader definition implies it also has a meaning of a mathematical operation on independent variable(s). Apart from acquiring the main concepts, the students learn to make decisions on what differentiation rules to use based on the decision tree (Fig 1) and the concept of the last operation in the function they are asked to differentiate. The decision tree summarises the steps needed to differentiate any analytical function. This is a scaffolding tool that can be discarded once the process has been internalised by a student.

For example, to differentiate $f(x)=\cos (2 x+3)$ students go down the decision tree and their attention is focussed on aspects of the differentiation process, which would otherwise be assumed to be done implicitly. The students need to think explicitly about the basics: "What function am I differentiating?", "What is the differentiation variable?" followed by "What differentiation rule do I apply?" The students need to reflect on the meaning of the mathematical symbols used to describe the function by asking "What is the last operation in $f(x)$ ?" The answer is "cos" and not "times". This point must be clarified by the teacher by re-emphasising the difference between algebraic and functional brackets, because many students confuse the two. They "see" the multiplication sign between cos and
$(2 x+3)$, If they do, they happily go ahead to remove the brackets and differentiate the sum $\cos 2 \mathrm{x}+\cos 3$.

The decision tree forces students to learn algorithmic and iterative approaches to problem solving: ask questions each step of the way and act depending on the answer. The benefit of the sequencing is in the emphasis on the process rather than the outcome, which can be either the answer or the completed homework exercise. It requires less memorising than standard approaches and as mentioned above, leads to deeper learning through mastering selfexplanations [7]. These can be taught and the Socratic dialogue, we propose, is the best means of achieving this end.

\section{The e-PACT development}

While the teaching methodology described above puts a great emphasis on explanation of abstract mathematical concepts, acquisition of mathematics skills requires students to do a reasonable number of exercises and have their understanding of concepts and deep-level reasoning skills reinforced every time they make a mistake. This part of the educational process can be automated with a Cognitive Tutor System, a piece of software containing an artificial intelligence component to track students' work and tailor its feedback and hints, which captures the expertise of a specialist in a particular domain, therefore creating an artificial expert.

Two most prominent and relevant systems of this nature are AUTOTUTOR that is designed to conduct a Socratic dialogue with freshers studying Newtonian mechanics or IT [2] and a Carnegie Cognitive Tutoring System [1] that employs

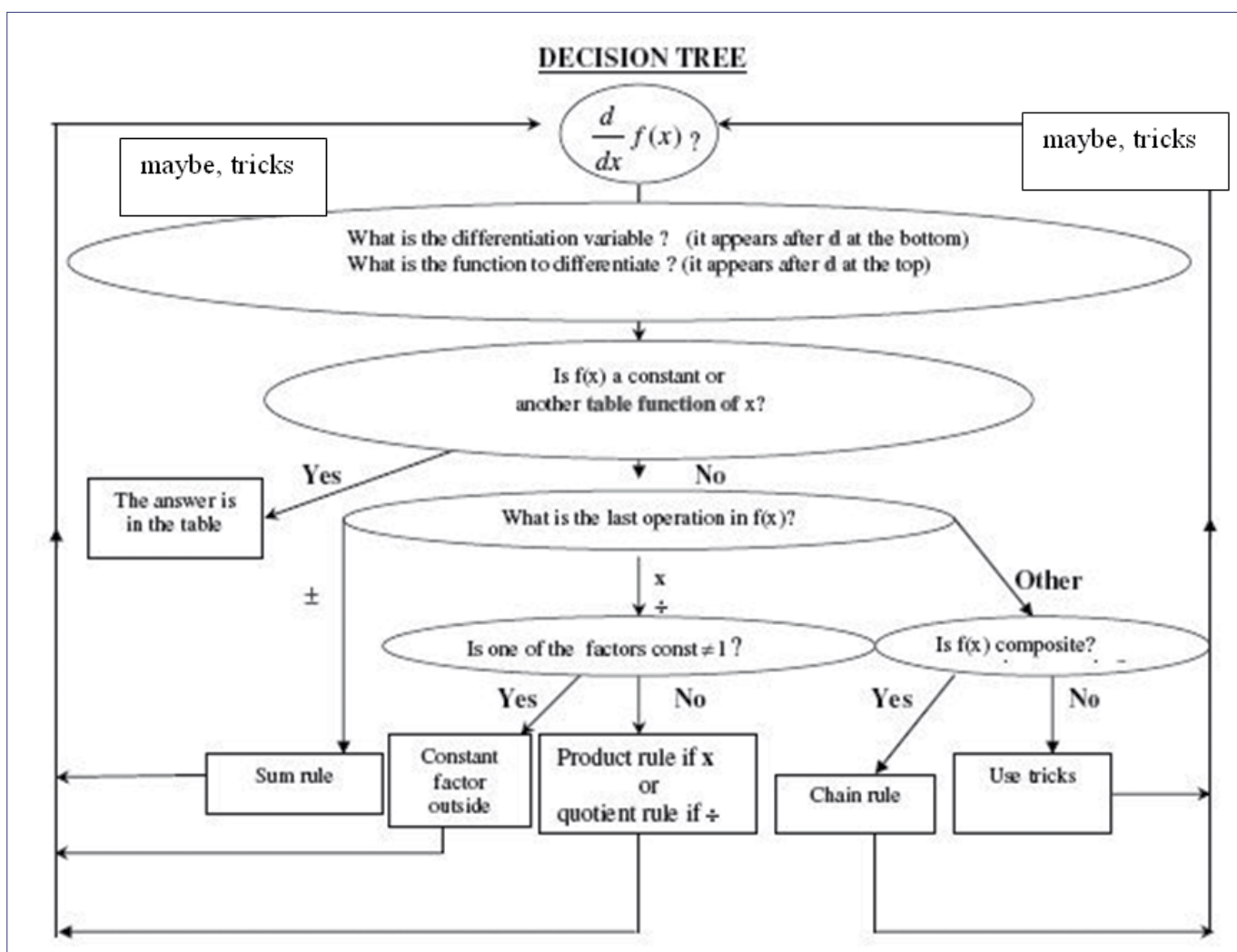

Fig 1 - Decision tree for differentiation 
a similar approach to teaching algebra and geometry in schools. We extend these ideas to more advanced algebra and calculus topics and produce the first Cognitive Mathematics Tutoring System, an electronic Personal Algebra and Calculus Tutor (e-PACT) (see Note 1), aimed at teaching basic mathematics at the University level. e-PACT is meant to allow users to practice their skills at their own pace. Importantly, there is no reliance on databases containing predefined problems and solutions. For each topic, on each level of difficulty, e-PACT is designed to generate at random a practically unlimited number of problems. If the user inputs an incorrect solution he/she will to be told that the solution is incorrect and be given a chance to input the correct one; on the second failed attempt a student receives an explanation of what aspects of his/her answer are wrong and on the third one, all mistakes and the corresponding corrections will be listed in full. The student will then be offered an explanation and if the offer is accepted, e-PACT would take him/her through the solution step by step using a Socratic dialogue based on Eulerian sequencing. In particular, in response to each of his/her inputs the user would receive a specific message generated by e-PACT which either asks what question should the user ask themselves now, or asks a question, or else gives a specific explanation of the question or feedback to an answer. Thus, e-PACT is designed to engage students in problem solving every step of the way, promotes deep-level reasoning via self-explanation and use decision trees that allow students to navigate through solution algorithms and formulae. As such, e-PACT is different to the host of interactive computer mathematics tutors available around the world which only offer digitised excerpts from standard textbooks to help learners deal with their mistakes.

At present, only the Differentiation Tutor has been implemented. A number of FESBE students were observed by independent researchers using the software. Despite being exposed to it for the first time, they needed very little assistance with the technical aspects of communicating with it, and even less assistance with the mathematics. They found e-PACT easy to use, since it follows the approach adopted in their lectures, tutorials and Blackboard material.

\footnotetext{
"It doesn't tell you what to do", one student said, and then went on to explain that the software does not show the next step in the solution or the answer, instead "the feedback encourages you to think about what you need to do; it takes you back to the decision tree, so you will be able to differentiate any function".
}

When he started the session with the software, another student was struggling with the level 1 questions (differentiation of elementary functions using the differentiation table). When feedback was prompted, he was not sure what the meaning of the word 'variable' was, but he was able to check that using the in-built glossary, which explained the word in detail, with specific examples. After a slow start using e-PACT, the student was then able to move up the levels and differentiate confidently more complex functions.

e-PACT records student sessions, and it is thus a very useful tool for a teacher to monitor the student learning and identify common mistakes or misconceptions.

\section{Conclusions}

Combining both Socratic questioning and Eulerian sequencing, the LSBU methodology is adapted to modern times to teach mathematics to engineering undergraduates, mostly with very poor mathematical background, about $26 \%$ of whom may be dyslexic, dyspraxic or suffer from dyscalculia.

The use of e-PACT, the Cognitive Tutor based on this methodology has the potential to transform the quality of learning and teaching, by providing students with an opportunity to practice their mathematical skills at their own pace but "under supervision" of an expert system. Providing a highly personalised feedback, it achieves a better match with learners' needs and dispositions and thus augments individual cognition. e-PACT provides an extra resource for students to enhance their mathematical skills, deepen their appreciation of mathematical concepts and improve their exam pass rates without additional teacher involvement, thus achieving higher quality and more effective learning in affordable and acceptable ways. Since it incorporates the novel textbook material and shows what questions can be asked of students at various stages to help them to master the material, it has the potential, for little or no extra cost, to be used as an aid in teacher training.

The methodology empowers learners with minor disorders who often have good engineering abilities but are known to have difficulties with mathematics. LSBU experience has shown that they can succeed with the Euler approach, because its systematic nature holds for them a particular appeal. Also, when constantly encouraged to watch the order and meaning of symbols such learners improve their performance to a remarkable degree and e-PACT can be of great help to speed up the progress.

Finally, the methodology ensures that students develop correct study skills, are taught rather than trained, thus mastering algorithmic and iterative approaches to problem solving and last but not least, learn the art of technical debate. 


\section{References}

1. Carnegie Learning System http://www. carnegielearning.com Pittsburgh, PA | 1-888.851-7094, U.K.

2. Chi, M.T.H. and VanLehn, K. A. (1991). The Content of Physics Self-Explanations, Journal of Learning Sciences, 1 (1), 69-105; AutoTutor, http://www.autotutor.org/

3. Collins (1985). Teaching reasoning skills. In S. Chipman, J. Segal, \& R. Glaser (Eds.). Thinking and Learning Skills. Vol. 2. (pp. 579-586). Hillsdale, NJ: Erlbaum.

4. Craig, S. D., Sullins, J., Witherspoon, A. and Gholson, B. (2006). The Deep-Level-Reasoning-Question Effect: The Role of Dialogue and Deep-Level-Reasoning Questions During Vicarious Learning. Cognition and Instruction, 4 (4), 565-591.

5. Hake, R.R. (1998) Interactive-engagement vs. traditional methods: A six-thousand-student survey of mechanics test data for introductory physics courses, American Journal of Physics. 66, 64.

6. Parson, S. (2004). Engineering Mathematics and the Dyslexic Student, presentation on 16 March 2004. Available via: http://www.wiziq.com/tutorial/698-EngineeringMathematics-and-the-Dyslexic-Student [Accessed 17 July 2009].

7. Smith, A. (2004) Making Mathematics Count, Report of Professor Adrian Smith's Inquiry into Post-14 Mathematics Education, The Stationery Office Limited.

\section{Notes}

1 The current version of e-PACT is accessible via the website e-PACT.org (under development). A stand-alone version can be requested by emailing Prof Fradkin at fradkil@Isbu.ac.uk. 